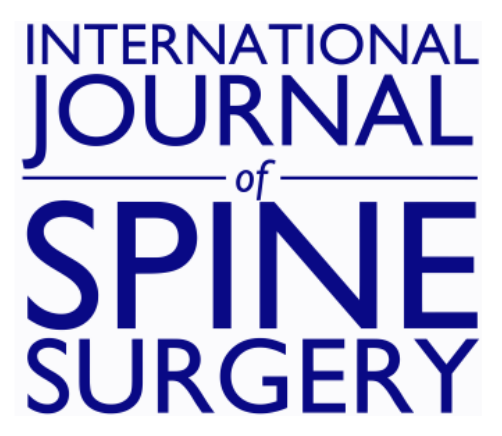

\title{
Lumbar Intervertebral Spacer With Cement Augmentation of Endplates and Integrated Screws as a Fixation Device in an Osteoporotic Model: An In Vitro Kinematic and Load-to-Failure Study
}

Rayshad Oshtory, Jonathan A. Harris, Pavan D. Patel, Belin A. Mirabile and Brandon S. Bucklen

Int J Spine Surg 2021, 15 (2) 324-333

doi: https://doi.org/10.14444/8042

http://ijssurgery.com/content/15/2/324

This information is current as of April 26, 2023.

Email Alerts Receive free email-alerts when new articles cite this article. Sign up at:

http://ijssurgery.com/alerts

The International Journal of Spine Surgery

2397 Waterbury Circle, Suite 1,

Aurora, IL 60504, Phone: +1-630-375-1432 


\title{
Lumbar Intervertebral Spacer With Cement Augmentation of Endplates and Integrated Screws as a Fixation Device in an Osteoporotic Model: An In Vitro Kinematic and Load-to-Failure Study
}

\author{
RAYSHAD OSHTORY, MD, MBA, ${ }^{1}$ JONATHAN A. HARRIS, MS, ${ }^{2}$ PAVAN D. PATEL, BS, ${ }^{3}$ \\ BELIN A. MIRABILE, BS, ${ }^{4}$ BRANDON S. BUCKLEN, PHD ${ }^{2}$ \\ ${ }^{I}$ Pacific Heights Spine Center, San Francisco, California, ${ }^{2}$ Musculoskeletal Education and Research Center (MERC), a Division of Globus Medical, Inc, \\ Audubon, Pennsylvania, ${ }^{3}$ School of Biomedical Engineering, Science, and Health Systems, Drexel University, Philadelphia, Pennsylvania ${ }^{4}$ Department of \\ Aerospace and Mechanical Engineering, University of Notre Dame, Notre Dame, Indiana
}

\begin{abstract}
Background: Integrated lateral lumbar interbody fusion (LLIF) devices have been shown to successfully stabilize the spine and avoid complications related to posterior fixation. However, LLIF has increased subsidence risk in osteoporotic patients. Cement augmentation through cannulated pedicle screws enhances pedicle fixation and cageendplate interface yet involves a posterior approach. Lateral application of cement with integrated LLIF fixation has been introduced and requires characterization. The present study set out to evaluate kinematic and load-to-failure properties of a novel cement augmentation technique with an integrated LLIF device, alone and with unilateral pedicle fixation, compared with bilateral pedicle screws and nonintegrated LLIF (BPS + S).

Methods: Twelve specimens (L3-S1) underwent discectomy at L4-L5. Specimens were separated into 3 groups: (1) BPS + S; (2) polymethyl methacrylate (PMMA) augmentation, integrated LLIF, and unilateral pedicle screws (PMMA + UPS + iS); and (3) PMMA and integrated LLIF (PMMA + iSA) without posterior fixation. Flexion-extension, lateral bending, and axial rotation were applied. A compressive load was applied to L4-L5 segments until failure. An analysis was performed $(P<.05)$.

Results: Operative constructs significantly reduced motion relative to intact specimens in all motion planes $(P<$ $.05)$. BPS + S provided the most stability, reducing motion by $71.6 \%-86.4 \%$, followed by PMMA + UPS + iS $(68.1 \%-$ $79.4 \%)$ and PMMA + iSA $(62.9 \%-81.9 \%)$; no significant differences were found $(P>.05)$. PMMA + UPS + iS provided the greatest resistance to failure $(2290 \mathrm{~N})$, followed by PMMA + iSA $(1970 \mathrm{~N})$ and BPS + S (1390 N); no significant differences were observed $(P>.05)$.

Conclusions: Cement augmentation of vertebral endplates via the lateral approach with integrated LLIF moderately improved cage-endplate strength compared to BPS $+\mathrm{S}$ in an osteoporotic model; unilateral pedicle fixation further improved failure load. Reconstruction before and after application of unilateral pedicle screws and rods was biomechanically equivalent to anteroposterior reconstruction. Overall, initial results suggest that integrated LLIF with cement augmentation may be a viable alternative in the presence of osteoporosis.
\end{abstract}

Biomechanics

Keywords: lateral interbody fusion, biomechanics, lumbar fusion, osteoporosis, bone cement, load to failure

\section{INTRODUCTION}

Interbody arthrodesis with supplemental bilateral pedicle screws (BPS) and rods is commonly used to treat destabilizing segmental pathologies, such as degenerative disc disease, spondylolisthesis, and deformity. While anteroposterior reconstruction immobilizes the segment across all three columns, ${ }^{1,2}$ paraspinal dissection and blood loss, ${ }^{3}$ significant financial cost, ${ }^{4}$ and iatrogenic infection ${ }^{5,6}$ associated with pedicle screw fixation remain disadvantages. The incidence of chronic spinal pathology increases with age, ${ }^{7}$ as do rates of major complications during elective surgery for elderly patients. Carreon et $\mathrm{al}^{8}$ report major and minor complication rates of $21 \%$ and $70 \%$, respectively, and $10 \%$ wound infection rates for patients undergoing lumbar decompression and arthrodesis, noting a significant positive correlation of complication rates with older age, blood loss, and longer operative time.

Minimally invasive, integrated lateral lumbar interbody fusion (LLIF) has been proposed to reduce approach-related morbidity in comparison to tradi- 
tional posterior spinal surgery. ${ }^{9-11}$ LLIF offers several advantages when compared with anterior or posterior interbody approaches, including preservation of the anterior longitudinal ligament, ${ }^{11}$ the ability to span the denser cortical ring apophysis, ${ }^{12}$ and decreased risk of injury to the great vessels. LLIF reconstruction is less invasive (1 incision) than anteroposterior lumbar fusion with BPS (up to 5 incisions) and may decrease the complications associated with BPS and accelerated by age. Clinically, integrated LLIF provides satisfactory patient outcomes, with reported fusion rates between $86.5 \%$ and $95 \%$ of levels treated. ${ }^{9,10}$

Despite the aforementioned benefits, subsidence rates of LLIF devices between $10 \%$ and $30 \%$ are reported. ${ }^{13,14}$ Contraindications, such as low bone mineral density (BMD) ${ }^{15}$ and older age, ${ }^{16}$ can further exacerbate postoperative cage subsidence, resulting in loss of disc height, reduced effect of indirect decompression, and recurrence of neurological deficits. ${ }^{13}$ Current techniques exist to increase the fixation strength in the osteoporotic spine, including laminar hooks, sublaminar tethering, and bone cement, but all act primarily to supplement posterior screw fixation. Bone cement has been shown to enhance fixation in suture anchor, trauma, and spine applications. Although polymethyl methacrylate (PMMA) bone cement has been traditionally used to enhance pedicle fixation, anterior PMMA augmentation - in kyphoplasty or vertebroplasty procedures - has been clinically shown to fortify endplates and prevent cage subsidence. ${ }^{17}$ Taken in combination with the available biomechanical literature, ${ }^{18}$ these initial results indicate that PMMA augmentation of the vertebral endplates may be sufficient in preventing subsidence of standalone LLIF reconstructions in older, osteoporotic patients.

The present study aimed to introduce a novel technique of cement augmentation of the vertebral endplates in conjunction with an integrated LLIF spacer before and after being supplemented with unilateral pedicle fixation and to evaluate the kinematic and compressive load-to-failure properties of the proposed techniques in comparison with anteroposterior fixation of traditional BPS and a nonintegrated LLIF interbody spacer.

\section{MATERIALS AND METHODS}

\section{Specimen Preparation}

Twelve fresh frozen osteoligamentous human cadaveric lumbosacral specimens (L3-S1) were used in this investigation with the operative level at L4L5. The medical history of each donor and standard anteroposterior and lateral plain films were reviewed to exclude any specimens with pathologies that would otherwise affect the outcome of testing. Dual-energy x-ray absorptiometry scans to evaluate the BMD (reported as $\mathrm{T}$ score) were performed using a water-bath protocol $^{19}$ for the lumbar segment in the coronal plane with a Lunar Prodigy 8743 scanner (GE Medical Systems, Waukesha, Wisconsin). Lumbosacral spines were thawed to room temperature and carefully denuded of paraspinal musculature, leaving only ligaments, bones, and intervertebral discs of the segments desired. Segments were potted at L3 and the sacrum using a 1:1 mixture of Bondo auto filler (Bondo MarHyde Corp, Atlanta, Georgia) and fiberglass resin (Home Solution All-Purpose, Bondo MarHyde Corp). All specimens were double wrapped in plastics bags and stored at $-20^{\circ} \mathrm{C}$ until testing.

\section{Operative Constructs}

A total of 12 specimens were divided into 3 equal groups ( $\mathrm{n}=4$ spines) such that the average BMD was similar between groups. Three treatment options were tested to investigate the differences in range of motion (ROM) and compressive load to failure of (1) traditional LLIF with supplemental bilateral pedicle screws and rods (BPS + S), (2) PMMA vertebral endplate augmentation and integrated lateral interbody spacer with unilateral pedicle screws and rod ipsilateral to the LLIF (PMMA + UPS + iS), and (3) PMMA vertebral endplate augmentation and integrated lateral interbody spacer (PMMA + iSA) without posterior fixation (Figure 1). Within each group, ROM was captured (1) for the intact condition and (2) following operative reconstruction. Surgical constructs included 6.5-mm-diameter titanium (Ti) bilateral pedicle screw fixation and 5.5-mm-diameter Ti rods (REVERE Stabilization System, Globus Medical, Inc, Audubon, Pennsylvania). Lateral interbody reconstruction used TransContinental and integrated InterContinental systems (Globus Medical, Inc). CONCORD (Globus Medical, Inc) bone cement was used for PMMA augmentation. Pedicle screw length was determined through probing of the pedicle. Interbody spacer heights were selected to achieve approximately $1 \mathrm{~mm}$ of distraction. Interbody spacers were $22 \mathrm{~mm}$ wide with $6^{\circ}$ of lordosis to maximize contact with the ring 


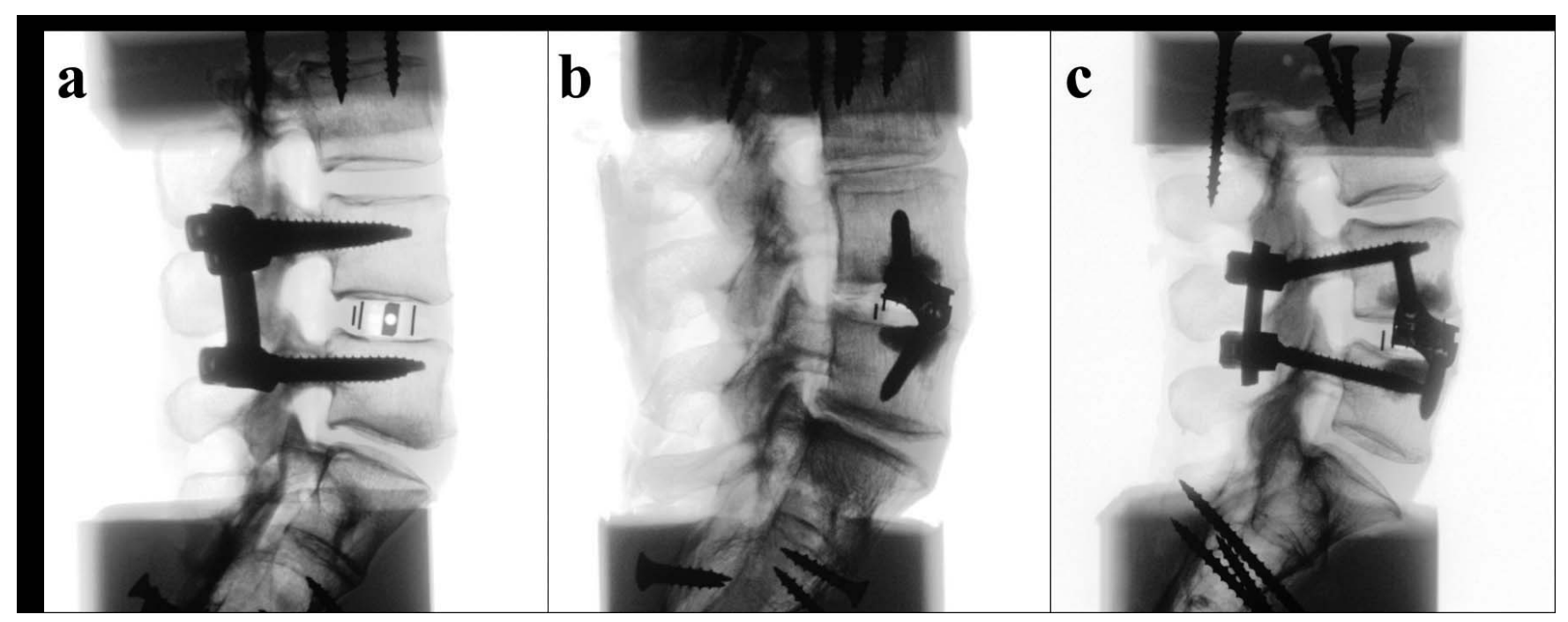

Figure 1. Representative sagittal fluoroscopic image of investigated anterior lumbar constructs. A. BPS $+S$; B. PMMA+iSA; C. PMMA+UPS+iS. BPS, bilateral pedicle screws; iS, integrated lateral lumbar interbody spacer; iSA, integrated lateral lumbar interbody spacer without posterior fixation; PMMA, polymethyl methacrylate; S, lateral lumbar interbody spacer; UPS, unilateral pedicle screws.

apophysis and thus maximize load to failure. ${ }^{1,12}$ Spacer lengths were determined such that the spacer spanned the ring apophysis. Integrated LLIF constructs used 5.5-mm-diameter Ti vertebral body screws with lengths determined using coronal radiography.

\section{Surgical Technique}

Following intact testing and pedicle screw fixation (when appropriate), adjacent endplates of the L4 and L5 vertebral bodies in the PMMA + UPS + iS and PMMA + iSA groups were identified using lateral radiographs. A 10-gauge, quad-tipped vertebroplasty needle (Globus Medical) was inserted into the vertebral bodies (patient's left, ipsilateral to discectomy) at the intersection point between the vertical midline and the horizontal line approximately $3 \mathrm{~mm}$ (thickness of the vertebroplasty needle) deep from the endplate. After puncturing the lateral cortical shell, the needle was progressed through the cancellous bone until impeded by the contralateral wall, as confirmed using coronal fluoroscopy and tactile feedback. The needle was repeatedly inserted at different trajectories through the same insertion point so that a cavity was created along the same plane deep to the endplate, which was necessary for even distribution of the bone cement. The radiopaque copolymer bone cement was prepared by mixing together the liquid and powder components for approximately 40 seconds. Mixing time was experimentally determined prior to testing in the present study to obtain appropriate viscosity. The prepara- tion of bone cement as instructed by manufacturer specifications (60 seconds) increased cement viscosity and impeded diffusion of cement through the intraoperative defect created deep to the endplate.

Approximately $3 \mathrm{cc}$ of PMMA was injected into each vertebral body using a 1.5-cc filler delivery needle and plunger, first toward the contralateral cortical shell and then filling near the point of entry. The described application technique consistently resulted in a cement mass limited to approximately the $25 \%$ of the vertebral body adjacent to the operative endplate. This cement mass projected through the entire width of the vertebral body, as confirmed by anteroposterior and lateral fluoroscopy. Representative axial radiographs of L4 and L5, disarticulated following compression testing, illustrate distribution of bone cement achieved (Figure 2). As recommended by the manufacturer, bone cement

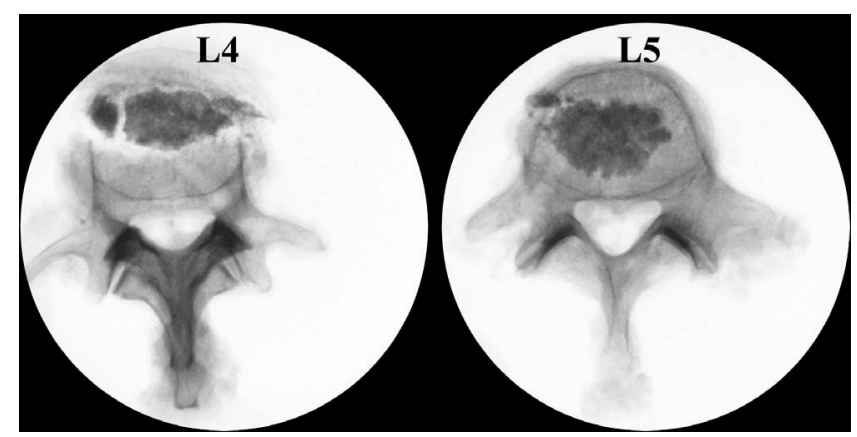

Figure 2. Representative axial fluoroscopic image of bone cement distribution of disarticulated L4 (left) and L5 (right) following compressive testing. Note that the vertebral body fracture at $L 4$ and cement fragmentation at L5 resulted from failure testing, not the initial application of cement. 
was left to cure for 20 minutes prior to endplate preparation to avoid potential leakage into the disc space during bilateral release of the annulus fibrosis and insertion of the lateral interbody device. ${ }^{17}$ No cases of bone cement leakage into the disc space or out of the point of entry were observed. Following placement of the lateral interbody spacer, screw trajectories were created with a straight awl. A 5.5$\mathrm{mm}$-diameter drill and tap were used to purchase the bone cement.

\section{In Vitro Biomechanical Testing}

\section{Multidirectional Testing}

Each specimen was thawed overnight and affixed to a custom-built 6-degrees-of-freedom testing apparatus used to simulate physiological loads. The cranial and caudal portions of the spinal segments were affixed to a 6-degrees-of-freedom motor gimbal assembly that applied a pure, unconstrained rotational moment independently about the x-, y-, and z-axes corresponding to flexion-extension, axial rotation, and lateral bending. The gimbal assembly is attached to the test platform, which includes linear air-bearing guide rails ( $\mathrm{x}$ - and $\mathrm{z}$-axes) and a pneumatic-controlled linear actuator (y-axis) enabling pure, unconstrained translation. A load control protocol with servomotors applied a pure moment caudally at a rate of $1.5^{\circ} \mathrm{s}$ to a maximum moment of $\pm 7.5 \mathrm{Nm} .{ }^{20}$ Each test construct experienced 3 load-unload cycles in flexion-extension (FE), lateral bending (LB), and axial rotation (AR). Data were collected on the third cycle to allow for the viscoelasticity of the tissue.

Plexiglass markers, each with 3 infrared lightemitting diodes, were secured rigidly to each vertebral level via bone screws to track motion with the motion analysis system (Optotrak Certus, Northern Digital, Inc, Waterloo, Ontario, Canada) placed approximately 6 feet in front of the specimen. Markers denoting a rigid body were aligned to approximate the sagittal curvature of the spine. The Optotrak Certus software superimposes the coordinate systems of two adjacent vertebral bodies to inferentially determine relative Eulerian rotations in each of the 3 planes with rotational and translational accuracy of $0.05^{\circ}$ and $0.03 \mathrm{~mm},{ }^{21}$ respectively, and resolution of $0.01 \mathrm{~mm} .^{22}$ Stability of the tested constructs was assessed through measurement of peak-to-peak ROM reported across the fixated level (L4-L5) and normalized to the average intact condition (mean intact $=100 \%$ ).
Table 1. Cadaveric specimen demographics (L3-S1) (average \pm SD).

\begin{tabular}{llll}
\hline Treatment Group & Sex (M, F) & \multicolumn{1}{c}{ Age (y) } & T Score \\
\hline BPS+S & 2 M, 2 F & $64.0 \pm 7.6$ & $-1.9 \pm 0.7$ \\
PMMA + UPS+ iS & $1 \mathrm{M}, 3$ F & $60.3 \pm 10.0$ & $-1.8 \pm 0.5$ \\
PMMA + iSA & $3 \mathrm{M}, 1 \mathrm{~F}$ & $65.8 \pm 9.6$ & $-1.8 \pm 0.6$ \\
\hline
\end{tabular}

Abbreviations: BPS, bilateral pedicle screws; iS, integrated lateral lumbar interbody spacer; iSA, integrated lateral lumbar interbody spacer without posterior fixation; PMMA, polymethyl methacrylate; S, lateral lumbar interbody spacer; UPS, unilateral pedicle screws.

\section{Ultimate Load-to-Failure Testing}

Following ROM testing, the L4-L5 functional spinal unit was resected and potted using a 1:1 mixture of Bondo auto filler and fiberglass resin to provide a flat surface for mechanical testing. Loadto-failure compression testing of the endplate was performed using a servohydraulic MTS 858 Bionix testing device (MTS Corp, Minneapolis, Minnesota; Figure 2). Uniaxial compression load-to-failure testing was conducted at a rate of $5 \mathrm{~mm} / \mathrm{s}$ to the point of failure. Load displacement data acquisition was acquired through an analog-to-digital DAS16G Metrabyte board (Metrabyte Corp, Taunton, Massachusetts) at a rate of $10 \mathrm{~Hz}$ to a personal computer. All data files were downloaded for spreadsheet computational data analysis. Peak load at endplate collapse and/or vertebral body fracture was recorded.

\section{Statistical Analysis}

Statistical analysis was performed by using IBM SPSS Statistics (version 24.0.0.0, IBM Corp, Armonk, New York). A paired $t$ test was performed to identify significant changes of intact motion following operative reconstruction per treatment group. Additionally, 1-way independent analysis of variance was performed to elucidate differences between treatment groups as assessed through BMD, ROM, and compressive load to failure. Significance was defined as $P<.05$.

\section{RESULTS}

A summary of treatment group demographics and BMD is presented in Table 1. No significant differences in BMD between treatment groups were observed $(P=.992)$.

\section{Multidirectional ROM}

A summary of raw and normalized ROM values (Tables 2 and 3), paired $t$ test $P$ values achieved comparing intact and treatment ROM with signif- 
Table 2. Raw L4-L5 motion, degrees (average \pm SD).

\begin{tabular}{|c|c|c|c|c|c|c|}
\hline \multirow[b]{2}{*}{ Mode } & \multicolumn{2}{|c|}{$\mathbf{B P S}+\mathbf{S}$} & \multicolumn{2}{|c|}{ PMMA + UPS + iS } & \multicolumn{2}{|c|}{ PMMA + iSA } \\
\hline & Intact & Treatment & Intact & Treatment & Intact & Treatment \\
\hline $\mathrm{FE}$ & $7.6 \pm 2.0$ & $1.3 \pm 0.3$ & $11.1 \pm 1.0$ & $3.4 \pm 1.7$ & $7.6 \pm 2.4$ & $2.6 \pm 1.4$ \\
\hline LB & $7.7 \pm 2.1$ & $1.1 \pm 0.4$ & $11.4 \pm 1.7$ & $2.4 \pm 0.3$ & $7.2 \pm 2.1$ & $1.3 \pm 1.5$ \\
\hline AR & $4.5 \pm 1.6$ & $1.3 \pm 0.3$ & $5.7 \pm 2.6$ & $1.8 \pm 0.8$ & $3.8 \pm 0.5$ & $1.4 \pm 0.5$ \\
\hline
\end{tabular}

Abbreviations: AR, axial rotation; BPS, bilateral pedicle screws; FE, flexion-extension; iS, integrated lateral lumbar interbody spacer; iSA, integrated lateral lumbar interbody spacer without posterior fixation; LB, lateral bending; PMMA, polymethyl methacrylate; S, lateral lumbar interbody spacer; UPS, unilateral pedicle screws.

icant relationships (Table 4), and ROM normalized to the average injured condition with significant relationships (Figure 3) are presented. In FE, BPS + $\mathrm{S}$ provided the greatest stability $(17.3 \%$ of intact motion), followed by PMMA + UPS + iS (30.6\%) and PMMA + iSA $(34.2 \%)$; all treatment options significantly reduced intact motion $(P=.006, P=$ .005 , and $P=.03$, respectively). While BPS $+\mathrm{S}$ provided the greatest stability $(13.6 \%)$ in LB, PMMA + iSA (18.1\%) marginally reduced motion to a greater extent than PMMA + UPS + iS $(20.6 \%)$; nevertheless, all treatment options significantly reduced intact motion $(P=.012, P=.001$, and $P=.01$, respectively). Finally, in AR, BPS $+\mathrm{S}$ provided the greatest stability $(28.4 \%)$, followed by PMMA + UPS + iS (31.9\%) and PMMA + iSA (37.1\%); all treatment options significantly reduced intact motion $(P=.03, P=.025$, and $P=.012$, respectively). Comparisons between treatment options revealed no significant differences in FE, LB, or $\operatorname{AR}(P=.257, P=.734$, and $P=.595$, respectively).

\section{Ultimate Load to Failure}

Representative plots of ultimate load-to-failure as a function of time (Figure 4), specimen-specific load to failure as a function of BMD (Figure 5), and average load to failure (Figure 6) are presented. PMMA + UPS + iS provided the greatest resistance to failure $(2290 \mathrm{~N})$, followed by PMMA + iSA (1970 $\mathrm{N})$ and then BPS $+\mathrm{S}(1390 \mathrm{~N})$. Comparisons between treatment options neared significant differ- ences in load to failure (main effect, $P=.074$; BPS + $\mathrm{S}$ vs PMMA $+\mathrm{UPS}+\mathrm{iS}, P=.065)$.

\section{DISCUSSION}

Nontraumatic segmental disc degeneration occurs following changes to the intervertebral disc mechanobiology as a result of natural aging. If conservative treatment does not address postural or neurological deficits, decompression and arthrodesis are sometimes required. Despite the wide range of indications for posterior screw and rod fixation, certain shortcomings remain, such as tissue dissection and associated pain and blood loss, ${ }^{3}$, risk of iatrogenic infection $\left(3.7 \%{ }^{5}-16.9 \%{ }^{6}\right.$ of patients), and risk of implant-related complications, such as screw fracture $\left(4.3 \%{ }^{23}-12.4 \%{ }^{24}\right.$ of patients), screw loosening ( $1 \%-15 \%{ }^{25}$ of nonosteoporotic patients), and pseudarthrosis $\left(20.7 \% \%^{2}-35 \%{ }^{27}\right.$ of procedures $)$.

Increased age of patients further compounds the risk of postoperative complications, making optimum treatment unknown. ${ }^{8,15,16}$ While alternative reconstruction methods, such as integrated LLIF, exist to reduce complications associated with posterior fixation, age and the concomitant degradation of bone quality limit the feasibility of these techniques. Tempel et $\mathrm{al}^{15}$ observed a subsidence rate of $24 \%$ ( 80 of 335 patients) following integrated lateral reconstruction, noting that patients with DEXA $T$ scores of less than -1.0 are at a much higher risk of developing graft subsidence and revision surgery.

Insufficient bone quality has led to the development of novel anterior applications of PMMA

Table 3. L4-L5 motion normalized to average intact condition, \% (average \pm SD).

\begin{tabular}{|c|c|c|c|c|c|c|}
\hline \multirow[b]{2}{*}{ Mode } & \multicolumn{2}{|c|}{$\mathbf{B P S}+\mathbf{S}$} & \multicolumn{2}{|c|}{ PMMA + UPS + iS } & \multicolumn{2}{|c|}{ PMMA + iSA } \\
\hline & Intact & Treatment & Intact & Treatment & Intact & Treatment \\
\hline FE & $100 \pm 26.2$ & $17.3 \pm 4.5$ & $100 \pm 9.3$ & $30.6 \pm 15.4$ & $100 \pm 31.8$ & $34.2 \pm 18.6$ \\
\hline LB & $100 \pm 26.7$ & $13.6 \pm 5.0$ & $100 \pm 15.3$ & $20.6 \pm 2.8$ & $100 \pm 28.5$ & $18.1 \pm 21.0$ \\
\hline AR & $100 \pm 35.2$ & $28.4 \pm 6.6$ & $100 \pm 45.4$ & $31.9 \pm 14.7$ & $100 \pm 13.2$ & $37.1 \pm 12.4$ \\
\hline
\end{tabular}

Abbreviations: AR, axial rotation; BPS, bilateral pedicle screws; FE, flexion-extension; iS, integrated lateral lumbar interbody spacer; iSA, integrated lateral lumbar interbody spacer without posterior fixation; LB, lateral bending; PMMA, polymethyl methacrylate; S, lateral lumbar interbody spacer; UPS, unilateral pedicle screws. 
Table 4. L4-L5 intact versus treatment motion: $P$ values of paired $t$ tests. ${ }^{\text {a }}$

\begin{tabular}{lccc}
\hline Mode & BPS + S & PMMA + UPS + iS & PMMA + iSA \\
\hline FE & $.006^{*}$ & $.005^{*}$ & $.03^{*}$ \\
LB & $.012^{*}$ & $.001^{*}$ & $.01^{*}$ \\
AR & $.03^{*}$ & $.025^{*}$ & $.012^{*}$ \\
\hline
\end{tabular}

Abbreviations: AR, axial rotation; BPS, bilateral pedicle screws; FE, flexionextension; iS, integrated lateral lumbar interbody spacer; iSA, integrated lateral lumbar interbody spacer without posterior fixation; LB, lateral bending; PMMA, polymethyl methacrylate; S, lateral lumbar interbody spacer; UPS, unilateral pedicle screws.

${ }^{\mathrm{a}}$ Asterisk denotes statistical significance: $P<.05$.

cement, including supplementing cervical bone screws in anterior cervical discectomy and fusion or intercorporal fusion procedures, ${ }^{28,29}$ cement backfill of screw tract following placement and removal of pedicle screws placed either posteriorly or laterally into the vertebral body ${ }^{18}$ (similar to the Texas Scottish Rite system for anterior scoliosis correction $^{30}$ ), and anterior vertebral cement augmentation in anterior lumbar interbody fusion. ${ }^{17}$ Retrospective analyses of 46 patients by Kim et al ${ }^{17}$ observed significant reduction in subsidence $(19.6 \%$ vs $5.2 \%)$ and vertebral body height loss $(10.7 \%$ vs $3.9 \%$ ) in patients with anterior lumbar interbody fusion and BPS following bone cement augmentation using a vertebroplasty needle insertion via the anterior median retroperitoneal approach. Two patients experienced greater than $10 \%$ loss of preoperative disc height despite anterior cement augmentation. However, it was noted that in both patients, the bone cement mass was located farther away from the endplate, emphasizing the importance of placing cement directly beneath the endplate to prevent cage subsidence.

To obviate the need for pedicle screw fixation and to avoid incidence of subsidence in patients with low

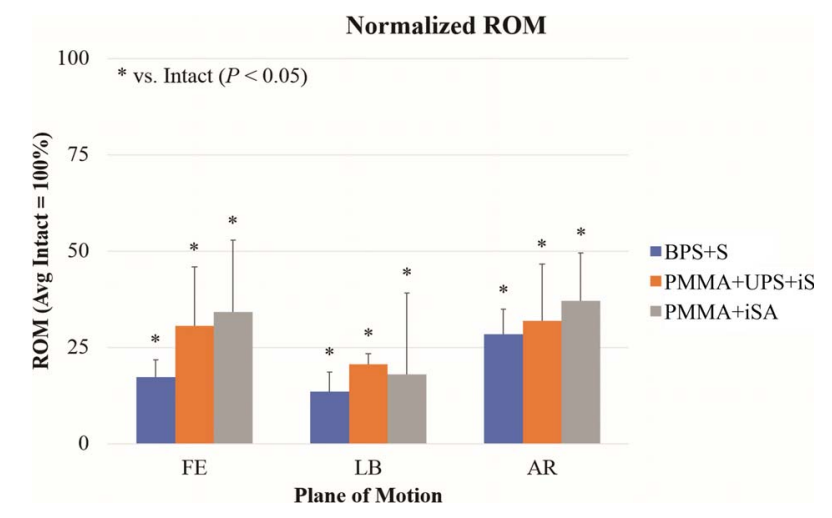

Figure 3. Range of motion at L4-L5 for treatment groups in all planes of motion. BPS, bilateral pedicle screws; is, integrated lateral lumbar interbody spacer; iSA, integrated lateral lumbar interbody spacer without posterior fixation; PMMA, polymethyl methacrylate; S, lateral lumbar interbody spacer; UPS, unilateral pedicle screws.

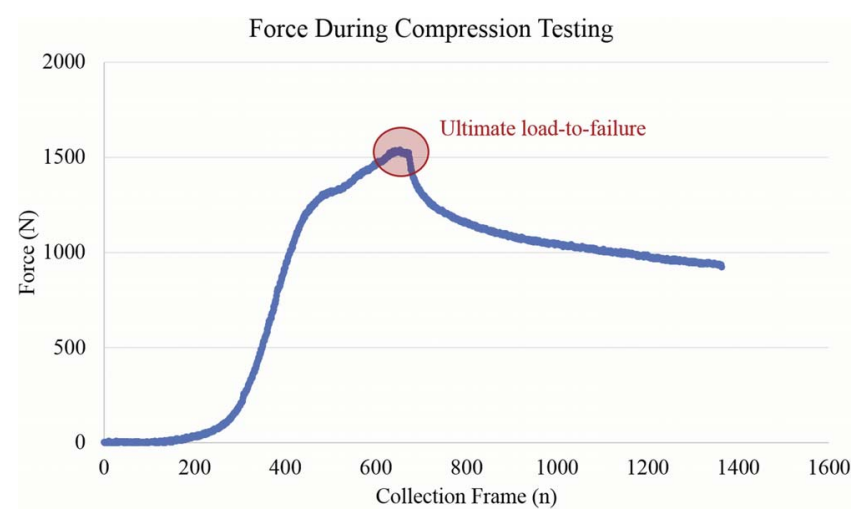

Figure 4. Representative load to failure as defined on force output from compression data.

BMD, the present study proposed a novel application of cement augmentation from a lateral approach adjacent to the endplates prior to implantation of an integrated LLIF device. The described technique would accommodate a single lateral transpsoas approach, enabling cement injection just under the adjacent endplates, insertion of an integrated plate spacer, and vertebral body screw fixation into the bone-cement interface. For posterior fixation, the lateral decubitus position of the patient allows unilateral pedicle screws to be inserted into the pedicles, ipsilateral likely being less difficult than contralateral.

Results of the present study found that all 3 proposed operative constructs significantly reduced intact motion. BPS $+\mathrm{S}$ provided the most stability in $\mathrm{FE}, \mathrm{LB}$, and $\mathrm{AR}(17.3 \%, 13.6 \%$, and $28.4 \%$ of intact ROM, respectively); however, comparisons with PMMA + UPS + iS $(30.6 \%, 20.6 \%$, and $31.9 \%$, respectively) or PMMA + iSA $(34.2 \%$, $18.1 \%$, and $37.1 \%$, respectively) were not found to

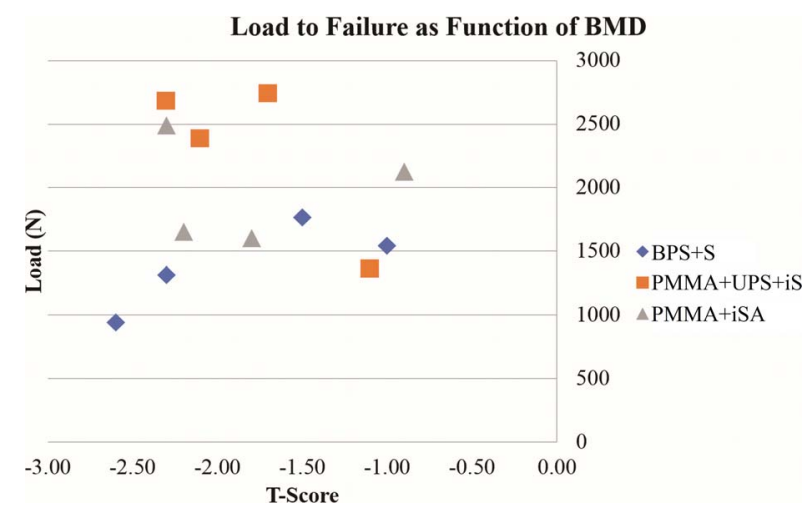

Figure 5. Ultimate load to failure as function of specimen specific bone mineral density per treatment group. BPS, bilateral pedicle screws; iS, integrated lateral lumbar interbody spacer; iSA, integrated lateral lumbar interbody spacer without posterior fixation; PMMA, polymethyl methacrylate; S, lateral lumbar interbody spacer; UPS, unilateral pedicle screws. 


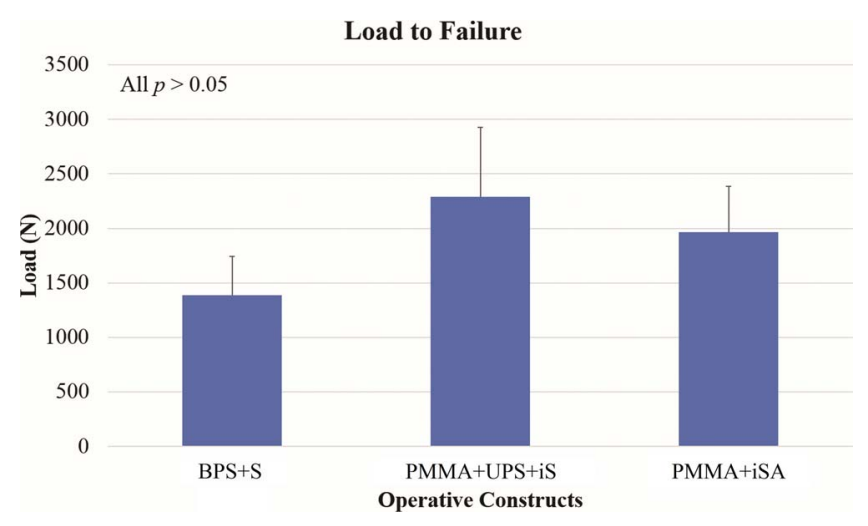

Figure 6. Ultimate load to failure per treatment group (mean $\pm \mathrm{SD}$ ). BPS, bilateral pedicle screws; iS, integrated lateral lumbar interbody spacer; iSA, integrated lateral lumbar interbody spacer without posterior fixation; PMMA, polymethyl methacrylate; S, lateral lumbar interbody spacer; UPS, unilateral pedicle screws.

be significant. Despite the application of bone cement, the observed trends are in general agreement with previous biomechanical investigations. Barsa et $\mathrm{al}^{31}$ found that BPS $+\mathrm{S}$ fixation provided the most fixation in FE, LB, and AR $(11.0 \%$, $16.1 \%$, and $26.9 \%$ of intact ROM, respectively), followed by UPS + iS $(14.1 \%, 24.2 \%$, and $39.8 \%$, respectively) and then iSA $(25.1 \%, 29.9 \%$, and $42.2 \%$, respectively); BPS $+\mathrm{S}$ significantly reduced motion in comparison to iSA in FE and AR $(P<$ $.05)$. The aforementioned results are roughly within the mean and standard deviations of the presented data; therefore, differences in significance may be due to random variations. Similar ROM results of the present study with Barsa et al, ${ }^{31}$ despite the addition of PMMA, suggest that bone cement augmentation of the endplates does not further stabilize the functional spinal unit regardless of engagement of the vertebral body screws with the cement.

Despite increased kinematic stability, BPS $+\mathrm{S}$ was found to provide the least resistance to subsidence. PMMA + UPS + iS and PMMA + iSA reconstruction increased ultimate load to failure in comparison to BPS $+\mathrm{S}$ by $64.7 \%$ (2290 vs 1390 $\mathrm{N}$, respectively) and $41.7 \%(1970 \mathrm{~N})$. These results are in broad agreement with previous compression analyses of anterior bone cement augmentation. Tan et $\mathrm{a}^{18}$ investigated the application of $2 \mathrm{cc}$ of PMMA through pedicle screw tracts after removal of a normally inserted screw, both along the traditional Weinstein approach and laterally through the vertebral body, as well as the failure load of both elliptical and cloverleaf-shaped indenters. Injecting PMMA along the posterior or lateral pedicle screw tract significantly increased mean failure load between $45 \%$ and $39 \%$ (averaged between the 2 indenter shapes) with mean failure loads between 1010 and 1920 N. Differences between cortical ring apophysis contact of LLIF spacers and elliptical or cloverleaf indenters, reported units of bone density, and operative constructs make comparisons of absolute load values difficult. Nevertheless, biomechanical results, in addition to initial clinical outcomes by Kim et al, ${ }^{17}$ suggest that anterior cement augmentation increases cage-vertebra interface strength and may reduce the incidence of subsidence.

The effectiveness of anterior cement augmentation to fortify the endplate and prevent cage subsidence depends critically on cement distribution. Traditional posterior applications of PMMA via cannulated pedicle screws offer 2 mediolateral injection sites but may not provide enough cement at the anterior region of the vertebral body to support the interbody device. $\mathrm{Hu}$ et $\mathrm{al}^{32}$ used computed tomography to evaluate cement distribution in the osteoporotic vertebral body across zone 1: the anterior third of the vertebral body; zone 2: the middle third of the vertebral body; zone 3: the posterior third of the vertebral body; and zone 4: the pedicle area. Of the 125 pedicle screws augmented with PMMA, $80 \%(n=100)$ of the screws resulted in cement distributed over 3 or 4 total zones; both the viscosity of the cement and the anterior position of the screw tip affected distribution. In the present study, a straight vertebroplasty needle was repeatedly inserted through a single entry point via the lateral approach in an effort to create a void adjacent to the endplate in zone 2, the final location of the integrated LLIF. Discectomy and placement of the interbody device directly above and below the PMMA layer resulted in compression of the endplate until the device contacted the cement during compression testing until fracture of either the cement mass or the vertebral body occurred. If the interbody device is not properly aligned with the cement mass (or if uneven distribution is seen in the coronal or sagittal plane), failure can occur along the path of least resistance. Gross photography following compression testing, as seen in Figure 7, shows the breakdown of the endplate. Once the interbody spacer contacted the bone cement, further axial load resulted in subsidence of the device's anterior edge into the endplate, where cement coverage was poor. 


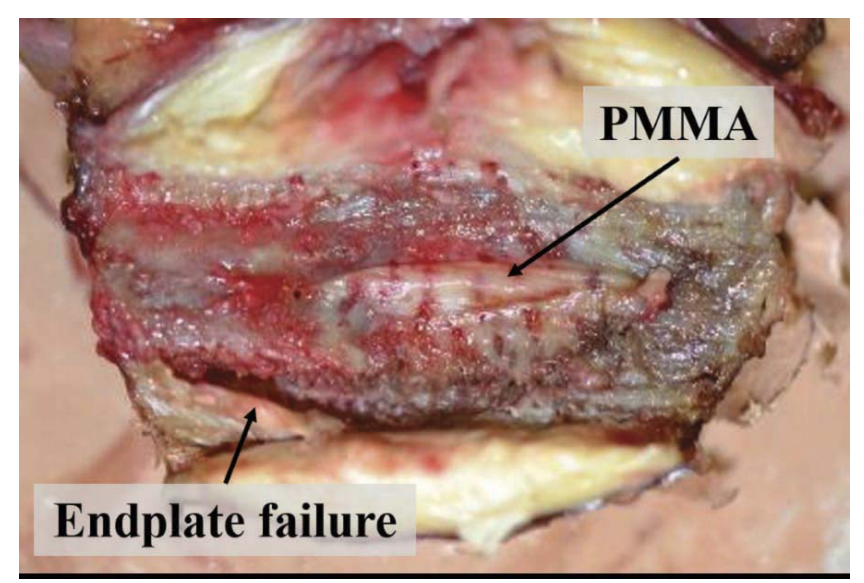

Figure 7. Representative gross photography of L4 endplate following ultimate load-to-failure testing. PMMA, polymethyl methacrylate.

Clinically, the surgeon author has performed lateral cement endplate augmentation using 9-inch Jamshidi needles inserted at the intersection point between the vertical midline and the horizontal line just deep to the endplate and advanced using a mallet. Starting at the far contralateral cortical shell, cement is injected while slowly withdrawing the needle in order to spread the cement evenly along the endplate. Typically, cement will have a more trabecular spread pattern in vivo than was observed in vitro during this study, potentially due to differences in BMD. The technique described in the present study, performed in the absence of vertebral fracture, is done solely to augment the endplates in an osteoporotic spine with the intention of minimizing implant subsidence and screw pullout. Clinically, the cement has always hardened by the time any screws are inserted; however, the surgeon author has not encountered any trouble inserting screws percutaneously through the injected cement, as the cement trabeculae are typically easily penetrated by the Jamshidi needle, tap, and screw (another reason a cavity is not created and filled), providing a hypothetically improved screw purchase.

The aim of anterior cement augmentation via the lateral approach and integrated LLIF is to offer a potential advantage over BPS in stabilizing the spinal segment without the additional incisions and paraspinal dissection. Nevertheless, appropriate indication and technique-related complications may be limitations. In the absence of supplemental pedicle fixation, the proposed technique depends solely on the distribution of cement. Patient selection is most critical if cement augmentation and interbody reconstruction are used. The risk associated with interbody fusion in the presence of poor bone quality may limit the indications of this technique. To maximize anterior distribution of the cement, the viscosity of PMMA was lowered by reducing the mixing time of the copolymers. Increasing diffusion by manipulating cement viscosity theoretically increases the risk of extravasation. ${ }^{33}$ Direct visualization of the operative levels provides confirmation of cement expulsion from the injection site; however, continuous fluoroscopy is advised.$^{34}$ Clinical literature additionally recommends maintenance of increased intrathoracic pressure throughout the procedure, intraosseous venography before cement injection, and postoperative fluoroscopy. ${ }^{35-37}$ Cement injection adjacent to the endplate, in an effort to minimize subsidence, ${ }^{17}$ carries the additional risk of extravasation into the disc space and may impede discectomy or insertion of the LLIF spacer.

While the presented work introduced a novel technique of cement augmentation of the vertebral endplates in conjunction with an integrated LLIF spacer and quantified the biomechanical efficacy of integrated LLIF before and after unilateral pedicle fixation in comparison with traditional anteroposterior reconstruction, this study is not without limitations. First, captured motion data reflect only the immediate postoperative condition and do not account for patient factors, such as bone healing and biomechanical features of the final fusion mass. It is within reason to expect the fusion bed formed across the anterior column to further augment the anterior-posterior loading dynamics of the construct. Anatomic differences between specimens could have affected results; however, all motion data were normalized to the intact state to limit this influence. Furthermore, mechanical properties, such as ligament laxity and quality of nucleus pulposus, may have been hindered by tissue desiccation. Although saline $(0.9 \%)$ was sprayed on the specimen throughout testing in an effort to preserve the connective tissues' viscoelastic properties, desiccation due to extended procurement time of the spine or time spent in the freezer before shipping could not be controlled. Finally, as is inherent in many cadaveric studies, a larger sample size may reduce the likelihood of type I (false-positive) and type II (false-negative) errors.

\section{CONCLUSIONS}

The current study introduced a novel technique of anterior cement augmentation of the adjacent 
endplates with integrated LLIF via a single-stage lateral approach to reduce complications associated with tissue dissection for pedicle screws and to address the risk of subsidence of interbody reconstruction in an osteoporotic model. All operative constructs significantly reduced intact motion. Bilateral pedicle screws with nonintegrated spacer provided the most stability in all planes of motion, followed by integrated LLIF with cement augmentation and unilateral posterior fixation; no significant differences were found between constructs. Cement augmentation of an integrated LLIF moderately increased failure load by $41.7 \%$ compared to bilateral pedicle fixation and spacer, and unilateral pedicle screws and rods further increased failure load to $64.7 \%$; however, no significant differences were found.

\section{REFERENCES}

1. Pimenta L, Turner AW, Dooley ZA, Parikh RD, Peterson MD. Biomechanics of lateral interbody spacers: going wider for going stiffer. Sci World J. 2012;2012:381814. doi:10.1100/2012/ 381814

2. Kornblum MB, Turner AW, Cornwall GB, Zatushevsky MA, Phillips FM. Biomechanical evaluation of stand-alone lumbar polyether-ether-ketone interbody cage with integrated screws. Spine J. 2013;13(1):77-84.

3. Kim DY, Lee SH, Chung SK, Lee HY. Comparison of multifidus muscle atrophy and trunk extension muscle strength: percutaneous versus open pedicle screw fixation. Spine (Phila Pa 1976). 2005;30(1):123-129.

4. Adogwa O, Parker SL, Davis BJ, et al. Cost-effectiveness of transforaminal lumbar interbody fusion for grade I degenerative spondylolisthesis. J Neurosurg Spine. 2011;15(2):138-143.

5. Klemencsics I, Lazary A, Szoverfi Z, Bozsodi A, Eltes P, Varga PP. Risk factors for surgical site infection in elective routine degenerative lumbar surgeries. Spine J. 2016;16(11):1377-1383.

6. Olsen MA, Nepple JJ, Riew KD, et al. Risk factors for surgical site infection following orthopaedic spinal operations. $J$ Bone Jt Surg Am. 2008;90(1):62-69.

7. Zigler JE, Capen DA, Rothman SL. Spinal disease in the aged. Clin Orthop Relat Res. 1995(316):70-79.

8. Carreon LY, Puno RM, Dimar JR, II, Glassman SD, Johnson JR. Perioperative complications of posterior lumbar decompression and arthrodesis in older adults. J Bone Jt Surg Am. 2003;85-A(11):2089-2092.

9. Ahmadian A, Bach K, Bolinger B, et al. Stand-alone minimally invasive lateral lumbar interbody fusion: multicenter clinical outcomes. J Clin Neurosci. 2015;22(4):740-746.

10. Marchi L, Abdala N, Oliveira L, Amaral R, Coutinho E, Pimenta L. Stand-alone lateral interbody fusion for the treatment of low-grade degenerative spondylolisthesis. Sci World J. 2012;2012:456346. doi:10.1100/2012/456346

11. Ozgur BM, Aryan HE, Pimenta L, Taylor WR. Extreme lateral interbody fusion (XLIF): a novel surgical technique for anterior lumbar interbody fusion. Spine J. 2006;6(4):435-443.
12. Briski DC, Goel VK, Waddell BS, et al. Does spanning a lateral lumbar interbody cage across the vertebral ring apophysis increase loads required for failure and mitigate endplate violation. Spine (Phila Pa 1976). 2017;42(20):E1158-E1164.

13. Le TV, Baaj AA, Dakwar E, et al. Subsidence of polyetheretherketone intervertebral cages in minimally invasive lateral retroperitoneal transpsoas lumbar interbody fusion. Spine (Phila Pa 1976). 2012;37(14):1268-1273.

14. Marchi L, Abdala N, Oliveira L, Amaral R, Coutinho E, Pimenta L. Radiographic and clinical evaluation of cage subsidence after stand-alone lateral interbody fusion. $J$ Neurosurg Spine. 2013;19(1):110-118.

15. Tempel ZJ, Gandhoke GS, Okonkwo DO, Kanter AS. Impaired bone mineral density as a predictor of graft subsidence following minimally invasive transpsoas lateral lumbar interbody fusion. Eur Spine J. 2015;24(suppl 3):414 419.

16. Hou Y, Luo Z. A study on the structural properties of the lumbar endplate: histological structure, the effect of bone density, and spinal level. Spine (Phila Pa 1976). 2009;34(12):E427-E433.

17. Kim KH, Lee SH, Lee DY, Shim CS, Maeng DH. Anterior bone cement augmentation in anterior lumbar interbody fusion and percutaneous pedicle screw fixation in patients with osteoporosis. J Neurosurg Spine. 2010;12(5):525-532.

18. Tan JS, Bailey CS, Dvorak MF, Fisher CG, Cripton PA, Oxland TR. Cement augmentation of vertebral screws enhances the interface strength between interbody device and vertebral body. Spine (Phila Pa 1976). 2007;32(3):334-341.

19. Tan JS, Kayanja MM, St Clair SF. The difference in spine specimen dual-energy X-ray absorptiometry bone mineral density between in situ and in vitro scans. Spine $J$. 2010;10(9):784-788.

20. Goel VK, Panjabi MM, Patwardhan AG, et al. Test protocols for evaluation of spinal implants. J Bone Jt Surg Am. 2006;88(suppl 2):103-109.

21. Maletsky LP, Sun J, Morton NA. Accuracy of an optical active-marker system to track the relative motion of rigid bodies. J Biomech. 2007;40(3):682-685.

22. Schmidt J, Berg DR, Ploeg HL. Precision, repeatability and accuracy of optotrak optical motion tracking system. Int $J$ Exp Comput Biomech. 2009;1(1):114-127.

23. Davne SH, Myers DL. Complications of lumbar spinal fusion with transpedicular instrumentation. Spine (Phila $\mathrm{Pa}$ 1976). 1992;17(suppl 6):S184-S189.

24. Jutte PC, Castelein RM. Complications of pedicle screws in lumbar and lumbosacral fusions in 105 consecutive primary operations. Eur Spine J. 2002;11(6):594-598.

25. Galbusera F, Volkheimer D, Reitmaier S, Berger-Roscher N, Kienle A, Wilke HJ. Pedicle screw loosening: a clinically relevant complication? Eur Spine J. 2015;24(5):1005-1016.

26. Koutsoumbelis S, Hughes AP, Girardi FP, et al. Risk factors for postoperative infection following posterior lumbar instrumented arthrodesis. J Bone Jt Surg Am. 2011;93(17):16271633.

27. Lonstein JE, Denis F, Perra JH, Pinto MR, Smith MD, Winter RB. Complications associated with pedicle screws. $J$ Bone Jt Surg Am. 1999;81(11):1519-1528.

28. Oppenlander ME, Bina R, Snyder LA, Dickman CA. Intravertebral polymethylmethacrylate augmentation of anterior cervical discectomy fusion and plating in the setting of osteoporosis. J Spinal Disord Tech. 2014;27(3):185-188.

29. Waschke A, Walter J, Duenisch P, Kalff R, Ewald C. Anterior cervical intercorporal fusion in patients with osteopo- 
rotic or tumorous fractures using a cement augmented cervical plate system: first results of a prospective single-center study. $J$ Spinal Disord Tech. 2013;26(3):E112-E117.

30. Turi M, Johnston CE II, Richards BS. Anterior correction of idiopathic scoliosis using TSRH instrumentation. Spine (Phila Pa 1976). 1993;18(4):417-422.

31. Basra S, Bucklen B, Muzumdar A, Khalil S, Gudipally M. A novel lateral lumbar integrated plate-spacer interbody implant: in vitro biomechanical analysis. Spine J. 2015;15(2):322-328.

32. Hu MH, Wu HT, Chang MC, Yu WK, Wang ST, Liu CL. Polymethylmethacrylate augmentation of the pedicle screw: the cement distribution in the vertebral body. Eur Spine J. 2011;20(8):1281-1288.

33. Choma TJ, Pfeiffer FM, Swope RW, Hirner JP. Pedicle screw design and cement augmentation in osteoporotic vertebrae: effects of fenestrations and cement viscosity on fixation and extraction. Spine (Phila Pa 1976). 2012;37(26):E1628-E1632.

34. Moreland DB, Landi MK, Grand W. Vertebroplasty: techniques to avoid complications. Spine J. 2001;1(1):66-71.

35. Groen RJ, du Toit DF, Phillips FM, et al. Anatomical and pathological considerations in percutaneous vertebroplasty and kyphoplasty: a reappraisal of the vertebral venous system. Spine (Phila Pa 1976). 2004;29(13):1465-1471.

36. Peh WC, Gilula LA. Additional value of a modified method of intraosseous venography during percutaneous vertebroplasty. Am J Roentgenol. 2003;180(1):87-91.

37. Yeom JS, Kim WJ, Choy WS, Lee CK, Chang BS, Kang JW. Leakage of cement in percutaneous transpedicular vertebroplasty for painful osteoporotic compression fractures. J Bone Jt Surg Br. 2003;85(1):83-89.

Disclosures and COI: The authors would like to disclose that this study was performed at Globus
Medical, Inc (GMI), using its 6-degrees-of-freedom motion simulator. R.O. is a consultant for GMI, Nuvasive, Alphatec, and Providence Medical Technologies, from which he receives teaching and consulting fees, and has provided expert testimony for Meridian MedLegal Management. Cadaveric specimens and related materials were provided by GMI, at which J.A.H. and B.S.B. are employees. P.D.P. and B.A.M. have nothing to disclose. The lateral integrated interbody spacer and bone cement examined in this study (InterContinental and CONCORD, respectively) are not approved by the US Food and Drug Administration for the application described within.

Corresponding Author: Rayshad Oshtory, MD, MBA, Pacific Heights Spine Center, 2100 Webster Street, Suite 314, San Francisco, CA 94115. Phone: (415) 737-0555; Fax: (415) 737-0595; Email: droshtory@phspine.com.

Published 16 April 2021

This manuscript is generously published free of charge by ISASS, the International Society for the Advancement of Spine Surgery. Copyright (c) 2021 ISASS. To see more or order reprints or permissions, see http://ijssurgery.com. 